\title{
From Theory to Practice: Engagement in Neurorehabilitation
}

Given patient and caregiver access to information through technology and the growing body of evidence indicating patient and caregiver engagement improves outcomes, gone are the days of the sage on the stage approach to healthcare in which the health professional reigns supreme. A simple internet search will yield a plethora of resources about patient engagement, from news articles to websites to conferences proceedings. This special edition seeks to advance the research in the area of patient and caregiver engagement so it becomes a mainstay upon which therapeutic relationships are founded and healthcare decisions in neurorehabilitation are made.

This issue includes contributions from a multidisciplinary group of investigators, representing fields including but not limited to social work, occupational therapy, speech language pathology, physical therapy, nursing and medicine. Patient and caregiver engagement is important to all and as such, contributions to this issue reflect an international body of work, including New Zealand, Australia, the United Kingdom and the United States. Given the complexities of engagement, contributions span research methods from quantitative to qualitative and address both the theoretical and the pragmatic applications and examinations of engagement. Engaging health professionals in addition to patients and caregivers is explored. Neurologic patient diagnoses include multiple sclerosis, stroke, and neuromuscular disorders.

From a background in home health care, Kenneth Miller articulates well the need for patient-centered care in today's world. A fundamental component of "patient-centered" care is patient engagement. Miller provides a review of theoretical underpinnings of engagement, including the trans-theoretical model of change, self-determination and motivational interviewing. Expanding on theory, Fiona Jones, Heide
Pöstges and Lucinda Brimicombe, of St Georges University of London and Kingston University, describe the Bridges methodology, an approach grounded in self-efficacy principles, to support self-management post-stroke in the United Kingdom. Their thoughtful discussion of the program inspires reflection about what engagement really means and avenues for how we, as health professionals, could do better.

Building on how healthcare providers can promote engagement, Maggie Bland and colleagues, from Washington University and Maryville University in Saint Louis, Missouri, provide a description and evidence for a clinical training model, Enhanced Medical Rehabilitation, aimed to promote patient engagement during therapeutic service delivery. While the model is not limited to neurorehabilitation, the approach, grounded in theories of behavior change, is applicable to any patient population. The program used in physical and occupational therapy sessions incorporates frequent feedback to patients, encouragement of patient autonomy and goal setting and an emphasis on developing the therapeutic connection with patients. Importantly, their work highlights the need for ongoing coaching efforts to health providers to improve adherence to methods to enhance engagement. A one-time training to clinicians is likely to fall short of changing practice. Just as patients require ongoing support to promote health behavior changes, so do clinicians to promote clinical practice changes. Providers will find their examples of conversations with patients to promote engagement a valuable starting point for reflecting on communication with patients.

Highlighting the importance of communication, work by Jessica Caron from the Pennsylvania State University focuses on increasing engagement through use of social media for individuals who use 
augmentative and alternative communication (AAC). After a review of an existing framework for consumer engagement on social media (including components of watching, sharing, commenting, producing and curating), Caron discusses its adaptability to increasing engagement with social media for individuals who use AAC.

Continuing our discussion of increasing patients' access to expressing themselves, work by Signal et al. and Travlos et al. bring the voices of those with neurological disorders to the discussion of engagement. Nada Signal and colleagues out of Auckland, New Zealand, examine patient engagement with high intensity exercise post-stroke. By examining facilitators and barriers identified by participants, a diverse group of individuals with stroke, this work identifies mediating factors that support engagement. Vivienne Travlos and colleagues from Perth, Western Australia examine perceptions of engagement in health, health behaviors, and healthcare by youth with neuromuscular disorders. The need for social, emotional, and physical support is clear, as is the need for a partnership with providers to support engagement.

Moving from qualitative research to a quantitative examination, Nora Fritz and colleagues from the United States posit that professionals can better facilitate engagement in neurorehabilitation provided a better understanding of the factors that mediate physical mobility. Given the heterogeneity of multiple sclerosis, exercise prescription and patient engagement can be challenging. Understanding the relationship of pain, quality of life, and cognition with structural measures of the brain can lead to individually tailored treatment planning and goal-setting, thereby improving patient engagement in neurorehabilitation.

The contributors to this special edition have a passion for improving the health and quality of life for those with neurological disorders. The authors advance an understanding of engagement and inspire critical reflection of engagement in clinical practice and research. It has been our privilege to work with them and we thank them for their dedication and commitment to advancing the art and science of engagement in neurorehabilitation. Now, the work of translating this knowledge into the classrooms of future providers, into clinical practice, and into future research begins.

\section{Acknowledgments}

To Professor Richard Andreatta and Professor Patrick Kitzman of the Rehabilitation Sciences program at the University of Kentucky in Lexington, Kentucky for inspiring a continued interest in patient and caregiver engagement in "Zan and Jayna" in the neuroplasticity course 5 years ago.

Guest Editors

Megan Danzl, PT, DPT, PhD, NCS

Assistant Professor

Department of Physical Therapy

Bellarmine University

Nolen C. Allen Hall

2001 Newburg Road

Louisville, KY 40205, USA

Tel.: +1 5022728356

E-mail: mdanzl@bellarmine.edu.

Nicole Etter, PhD, CCC/SLP

Assistant Professor

Department of Communication Sciences \&

Disorders

The Pennsylvania State University 404C Ford Building

University Park, PA 16802, USA

Tel.: +1 8148632021

E-mail: nme2@psu.edu. 